\title{
Metformin therapy for diabetes mellitus in Prader-Willi syndrome
}

\author{
A C D de Alwis ${ }^{1}$, A M R D Senarathne ${ }^{2}$, R N Morawakkorala ${ }^{2}$, B Weerasinghe ${ }^{3}$, S Liyanage ${ }^{4}$
}

Sri Lanka Journal of Child Health, 2007; 36: 24-26

(Key words: Metformin therapy, diabetes mellitus, Prader-Willi syndrome)

Prader-Willi syndrome (PWS) is a rare neurogenetic disorder, which occurs in 1 per 15,000 live-born children. First described in 1956 by doctors Prader, Labhart and Willi, it is the commonest cause of syndromic obesity in childhood. Diabetes mellitus $(\mathrm{DM})$ is a rare complication of children with PWS. A literature search revealed that most PWS children with DM have required insulin as their treatment while only a couple have responded to oral hypoglycaemic drugs. We report a 11 year old girl with PWS complicated by DM treated with the oral hypoglycaemic drug metformin.

\section{Case report}

An eleven year old girl from Weddagala presented to Ratnapura General Hospital with a three week history of polyuria, nocturia and polydipsia. She was born at term, weighing $1800 \mathrm{~g}$, to healthy non consanguineous parents following a pregnancy complicated by intrauterine growth retardation. As a newborn she was hypotonic with a feeble cry and feeding difficulties necessitating nasogastric feeding in the neonatal unit for almost a month. Cup and spoon feeding was continued at home for nearly four months by extra-caring parents. Weight gain was poor during the first two years of life but accelerated subsequently as a result of voracious appetite and obsessional overeating.

The developmental milestones were markedly delayed; she began to walk and grasp objects at 3 years and spoke the first clear words at 4 years. Currently she has moderate learning difficulties and special educational needs. In the absence of facilities for special education in her remote hamlet, she does not attend mainstream school but receives elementary education at home. Her appetite remains voracious; she takes five rice meals a day and craves for

${ }^{1}$ Consultant Paediatrician, ${ }^{2}$ Registrar, ${ }^{3}$ Senior House Officer, ${ }^{4}$ House Officer, General Hospital, Ratnapura.

(Received on 31 August 2006) sugar, sweets and biscuits. Feeling lethargic, she loves to sleep or to remain seated the whole day.

On examination, she was a cheerful, plump girl with a weight of $44 \mathrm{~kg}\left(75^{\text {th }}-91^{\text {st }}\right.$ centile $)$, height of $123 \mathrm{~cm}$ $\left(<0.4^{\text {th }}\right.$ centile $)$ and BMI of $31 \mathrm{~kg} / \mathrm{m}^{2}\left(>99.6^{\text {th }}\right.$ centile $)$. She has a narrow forehead, small, almond shaped palpebral fissures and small hands and feet. She was hypotonic, pre-pubertal and had a nasal voice. Blood pressure was 100/60 $\mathrm{mm} \mathrm{Hg}$ (normal for height and age) and examination of other systems revealed no abnormality.

At presentation, $2 \%$ of glycosuria without ketonuria and a high blood glucose concentration of $20.9 \mathrm{mmol} / \mathrm{L}$ were found. The blood urea was $17 \mathrm{mg} / \mathrm{dl}$, serum sodium $135 \mathrm{mEq} / \mathrm{L}$, serum potassium $4.1 \mathrm{mEq} / \mathrm{L}, \mathrm{Hb} 16 \mathrm{~g} / \mathrm{dl}$, white cell count $12 \times 10^{9} / \mathrm{L}$ with $52 \%$ neutrophils, AST $38 \mathrm{U} / \mathrm{L}$, ALT $29 \mathrm{U} / \mathrm{L}$, total cholesterol $166 \mathrm{mg} / \mathrm{dl}$, HDL cholesterol $43 \mathrm{mg} / \mathrm{dl}$, LDL cholesterol $101 \mathrm{mg} / \mathrm{dl}$, total cholesterol: HDL cholesterol 3.86, triglycerides 109 $\mathrm{mg} / \mathrm{dl}$ and $\mathrm{C}$ peptide $0.89 \mathrm{ng} / \mathrm{ml}$. Abdominal ultrasongraphy revealed fatty infiltration of the liver.

DM complicating PWS was diagnosed and soluble insulin $0.8 \mathrm{U} / \mathrm{kg} /$ day in three divided doses before breakfast, lunch and dinner started. On the $6^{\text {th }}$ day of treatment, when blood glucose concentration decreased to $12 \mathrm{mmol} / \mathrm{L}$, insulin was stopped and medication was switched over to oral metformin 250 $\mathrm{mg}$ twice daily. Dietary intake of calories was restricted to 2,200 kcal/day and a regular exercise programme was initiated. Dose of metformin was subsequently increased to $500 \mathrm{mg}$ twice daily.

She attained near-normal glycaemia a fortnight after starting metformin with fasting and post-prandial blood glucose readings of 3.3 and $8 \mathrm{mmol} / \mathrm{L}$ respectively. Three times daily testing for glycosuria at home revealed negative or $0.5 \%$ glucose in more than 90 percent of urine samples. Parents could not afford home blood glucose monitoring. Marked reduction of appetite and lowering of weight by $4 \mathrm{~kg}$ over 4 weeks was noticed at follow up clinic visit. 


\section{Discussion}

PWS is characterized by diminished fetal activity, muscular hypotonia, feeding difficulties and failure to thrive in infancy, hyperphagia and truncal obesity in childhood, short stature, hypogonadotrophic hypogonadism, moderate to severe learning difficulties, behavioural problems, almond shaped eyes and small hands and feet. It is considered as an autosomal dominant disorder and is caused by a deletion of a cluster of genes on the long arm of the paternal chromosome 15 or maternal uniparental disomy 15 because the gene(s) on the maternal chromosome(s) 15 are virtually inactive through imprinting ${ }^{1}$.

$\mathrm{DM}$ is becoming a more frequently recognized complication in adults with PWS. It has been reported that as many as $7-20 \%$ of individuals with PWS develop this complication ${ }^{2}$. The mean age of onset of DM is 20 years; hence it is rare in pre-pubertal children ${ }^{3}$.

The aetiology of DM in PWS is not fully understood. The initial assumption has been that the DM in this syndrome is identical to that seen in obese individuals without PWS, in whom genetic factors and obesity lead to insulin resistance. Severe insulin resistance in turn leads to pancreatic failure and hence the symptom complex of type 2 DM. But recent studies have demonstrated that individuals with PWS do not show the predicted insulin resistance that is seen in obese children without the syndrome. In fact, the individuals with PWS showed normal or increased insulin sensitivity disputing the hypothesis that the DM in PWS is simply the result of obesity ${ }^{2}$. Other studies have shown that non-diabetic PWS patients manifest a reduced beta-cell response to glucose stimulation and a significantly increased hepatic insulin extraction compared with obese controls ${ }^{4}$.

Management of DM in PWS include calorie restricted diet, regular exercise and anti-diabetic medication. Since most children with PWS exhibit obsessional overeating consequent to impaired satiety response, dietary restriction of calories has been difficult and unsuccessful. Many PWS children have needed antidiabetic medication. Insulin has been used in most cases while successful treatment with oral hypoglycaemic drugs is reported only in two children. Only one child treated with metformin is reported in the literature ${ }^{2,5}$.

Metformin, an oral hypoglycaemic drug belonging to the class of biguanides, is the drug of choice in the treatment of type $2 \mathrm{DM}$ in childhood ${ }^{6}$. It acts by increasing tissue sensitivity to insulin, principally in the liver via inhibition of hepatic glycogenolysis. Additional beneficial properties of metformin include a) weight reduction b) favourable effects on the lipid profile c) absence of hypoglycaemia and d) appetite reduction.
The weight reduction seen with metformin is in contrast to weight gain caused by insulin and sulphonylureas. Appetite reduction may be of particular significance in diabetes associated with overeating. Our patient achieved near-normal glycaemia and marked weight and appetite reduction with metformin. Lactic acidosis is a rare but important adverse effect of metformin which can occur in children with renal impairment. Hence metformin should be avoided in children with mild renal insufficiency and serum creatinine needs to be measured before and during treatment ${ }^{6,7}$.

This case illustrates the importance of considering the use of a relatively safe, cheap and easy to administer drug, metformin to treat the uncommon complication of $\mathrm{DM}$, in the rare genetic disorder, PWS.

\section{Acknowledgements}

We thank Dr Miss K S H de Silva, Senior Lecturer in Paediatrics, Faculty of Medicine, University of Colombo for her valuable help in management of this patient.

\section{References}

1. Prader-Willi syndrome; Online Mendelian Inheritance in Man, John Hopskin University website.

http;//www.ncbi.nih.gov/entrz/query/fcgi

last accessed in August 2006.

2. Zipf WB. Glucose homeostasis in Prader-Willi syndrome and potential implications of growth hormone therapy. Acta Paediatr Suppl. 1999; 88 (433): 115-7

3. Sills IN, Rapaport R. Non-insulin dependent diabetes mellitus in a prepubertal child with PraderWilli syndrome. J Pediatr Endocrinol Metab. 1998; 11(2):281.

4. Schuster DP, Osei K, Zipf WB. Characterization of alterations in glucose and insulin metabolism in Prader-Willi subjects. Metabolism. 1996; 45(12): 1514-20.

5. Chan NN, Feher MD, Bridges NA. Metformin therapy for diabetes in Prader- Willi syndrome $J R$ Soc Med 1998; 91(11):598.

6. Metformin; British National Formulary for Children 2005, British Medical Journal Publications Ltd.

7. Goodarzi MO, Bryer-Ash M. Metformin revisited: re-evaluation of its properties and role in the pharmacopoeia of modern antidiabetic agents. Diabetes Obes Metab. 2005; 7(6):654-6. 